Title:

Moment Characterization of Matrix Exponential and Markovian Arrival Processes

Authors:

Levente Bodrog

Department of Telecommunications, Technical University of Budapest

Andras Horvath

Dipartimento di Informatica, Università di Torino

Miklos Telek

Department of Telecommunications, Technical University of Budapest

Corresponding author:

Andras Horvath

Dipartimento di Informatica - Universit di Torino

Corso Svizzera 185 - 10149 Torino (Italy)

phone: +39-011-6706803

fax: $+39-011-751603$

e-mail: horvath@di.unito.it

Abstract:

This paper provides a general framework for establishing the relation between various moments of matrix exponential and Markovian processes. Based on this framework we present an algorithm to compute any finite dimensional moments of these processes based on a set of required (low order) moments. This algorithm does not require the computation of any representation of the given process. We present a series of related results and numerical examples to demonstrate the potential use of the obtained moment relations.

Keywords:

Matrix exponential process, Markov arrival process, Matrix exponential distribution, phase type distribution, inter-arrival time distribution, lag-correlation. 


\title{
Moment Characterization of Matrix Exponential and Markovian Arrival Processes*
}

\author{
Levente Bodrog ${ }^{2}$, András Horváth ${ }^{1}$, Miklós Telek $^{2}$ \\ ${ }^{1}$ Dipartimento di Informatica, Università di Torino, Italy \\ ${ }^{2}$ Dept. of Telecommunications, Technical University of Budapest, Hungary \\ E-mail: horvath@di.unito.it, \{bodrog,telek\}@webspn.hit.bme.hu
}

\begin{abstract}
This paper provides a general framework for establishing the relation between various moments of matrix exponential and Markovian processes. Based on this framework we present an algorithm to compute any finite dimensional moments of these processes based on a set of required (low order) moments. This algorithm does not require the computation of any representation of the given process. We present a series of related results and numerical examples to demonstrate the potential use of the obtained moment relations.

Keywords: Matrix exponential process, Markov arrival process, Matrix exponential distribution, phase type distribution, moment matching, inter-arrival time distribution, lag-correlation.
\end{abstract}

\section{Introduction}

Phase type (PH) distributions and Markovian arrival processes (MAP) are simple stochastic models that enjoy a simple stochastic interpretation based on Markov chains. They are widely used in traffic engineering because efficient numerical techniques are available for the solution of queueing models with PH distributions and MAPs $[1,2,3]$. Matrix exponential (ME) distributions and processes (MEP) are more general stochastic models than PH distributions and MAPs $[4,5]$. They do not have a simple stochastic interpretation and most of the methods applied to Markovian models cannot be directly applied to them. Still, in recent years we have seen a growing interest in these models and several results for queueing systems have been presented $[6,7,8,9]$.

To apply PH, ME distributions, MAPs and MEPs in stochastic models, we need good understanding of their properties. In this paper we investigate issues regarding the moments of these distributions and processes.

For what concerns ME distributions, a moment matching method is presented in [10] which is based on a partial realization algorithm published in [11]. Due to these works the behaviour of the moments of $\mathrm{PH}$ and ME distributions are known. In [12] a method is given to compute the order of a ME distribution

\footnotetext{
${ }^{*}$ This work is partially supported by the Italian-Hungarian bilateral R\&D programme, by OTKA grant n. T-34972, by MIUR through PRIN project Famous and by EEC project Crutial.
} 
based on different kinds of information on the probability distribution. In this paper, following a similar methodology as the one used in [12], we investigate through simple examples the connection between parameters (moments or derivatives of the cumulative density function) of the distribution and the moment matching problem.

On the other hand, for MAPs and MEPs, it is not known which are those moments and joint moments (we need joint moments because these are processes with correlated interarrival times) that determine the process. In [13] a procedure is presented which constructs a MEP based on the moments of the interarrival times and the lag-correlations. We show in this paper that these two sets of quantities do not determine the MEP, i.e., it is possible to construct different MEPs that are identical from the point of view of these parameters. In this paper we define a set of equations which relates the moments and joint moments of a MEP and define a minimal set of moments and joint moments based on which it is possible to determine any moment and joint moment of the process without knowing its representation.

The paper is organised as follows. Section 2 presents results for $\mathrm{PH}$ and ME distributions, while Section 3 deals with MAPs and MEPs. We formulate the results for ME distributions and MEPs, but they are directly applicable to PH distributions and MAPs as well. We conclude with Section 4.

\section{Matrix Exponential and Phase Type Distributions}

\section{$2.1 \quad$ Basic definitions}

Let $X$ be a continuous non-negative random variable with cumulative distribution function (cdf)

$$
F(t)=\operatorname{Pr}(X<t)=1-\alpha e^{A t} \mathbb{I},
$$

where row vector $\alpha$ is referred to as the initial vector, square matrix $A$ as the generator and $\mathbb{I}$ as the closing vector. Without loss of generality (see [14]), we assume that the closing vector, $\mathbb{I}$, is a column vector of ones. When the cardinality of the vectors and the square matrix is $n, X$ is referred to as an order $n$ matrix exponential distribution $(\mathrm{ME}(\mathrm{n}))$. As $X$ is a continuous random variable, it has no probability mass at zero, i.e., $\alpha \mathbb{I}=1$. The density, the Laplace transform, the moments and the reduced moments of $X$ can be computed as

$$
\begin{gathered}
f(t)=\alpha e^{A t}(-A) \mathbb{I}, \\
f^{*}(s)=E\left(e^{-s X}\right)=\alpha(s I-A)^{-1}(-A) \mathbb{I}, \\
\mu_{n}=E\left(X^{n}\right)=n ! \alpha(-A)^{-n} \mathbb{I} \\
r_{n}=E\left(X^{n}\right) / n !=\alpha(-A)^{-n} \mathbb{I} .
\end{gathered}
$$

In general, the elements of $\alpha$ and $A$ may be arbitrary real numbers. If $\alpha$ is a probability vector and $A$ is the generator matrix of a continuous-time Markov chain, then $X$ is an order $\mathrm{n}$ phase type distributed $(\mathrm{PH}(\mathrm{n})) . \alpha$ is a probability vector when $\alpha_{i} \geq 0(\forall i=1, \ldots, n)$ and $\alpha \mathbb{I}=1$ and matrix $A$ is a transient Markovian generator when $A_{i i}<0(\forall i=1, \ldots, n), A_{i j} \geq 0(\forall i, j=1, \ldots, n, i \neq j), A \mathbb{I} \leq \mathbf{0}, A \mathbb{I} \neq \mathbf{0}$.

To ensure that $f(t)$ in $(2)$ is a density function, $A$ has to fulfill the necessary but not sufficient condition that the real part of its eigenvalues are negative (consequently $A$ is non-singular). 
The vector together with the square matrix, $(\alpha, A)$, is referred to as the representation of the ME $(\mathrm{PH})$ distribution. In general, the $(\alpha, A)$ representation is not unique.

Definition 1. An $(\alpha, A)$ representation is non-redundant if the cardinality of vector $\alpha$ and square matrix $A$ is equal to the degree of the denominator of $f^{*}(s)$ (when the numerator and denominator of the function $f^{*}(s)$ are co-prime).

Throughout the paper we assume that the representations of $\mathrm{ME}(\mathrm{PH})$ distributions are nonredundant.

\subsection{Doubly infinite Hankel matrix}

We define the doubly infinite Hankel matrix as

$$
R=\left[\begin{array}{ccccccc}
\ddots & \vdots & \vdots & \vdots & \vdots & \vdots & \cdot \\
\cdots & r_{-4} & r_{-3} & r_{-2} & r_{-1} & r_{0} & \cdots \\
\cdots & r_{-3} & r_{-2} & r_{-1} & r_{0} & r_{1} & \cdots \\
\cdots & r_{-2} & r_{-1} & r_{0} & r_{1} & r_{2} & \cdots \\
\cdots & r_{-1} & r_{0} & r_{1} & r_{2} & r_{3} & \cdots \\
\cdots & r_{0} & r_{1} & r_{2} & r_{3} & r_{4} & \cdots \\
\cdot & \vdots & \vdots & \vdots & \vdots & \vdots & \ddots
\end{array}\right]
$$

where $r_{i}=\alpha(-A)^{-i} \mathbb{I}$.

For $i \geq 0, r_{i}$ is the $i$ th reduced moment, while for $i<0, r_{i}$ is related to the $i$ th derivative of the cdf, since the $i$ th derivative of the cdf of the ME distribution given by $\alpha$ and $A$ at $t=0$ is

$$
\left.\frac{d^{i} F(t)}{d t^{i}}\right|_{t=0}=-\alpha A^{i} \mathbb{I}=(-1)^{i+1} \alpha(-A)^{i} \mathbb{I}=(-1)^{i+1} r_{-i}
$$

Theorem 1. Under the non-redundant condition the rank of $R$ is $n$.

Proof. $R$ can be expressed as

$$
R=\left[\begin{array}{c}
\vdots \\
\alpha(-A)^{2} \\
\alpha(-A)^{1} \\
\alpha \\
\alpha(-A)^{-1} \\
\alpha(-A)^{-2} \\
\vdots
\end{array}\right]\left[\begin{array}{lllllll}
\cdots & (-A)^{2} \mathbb{I} & (-A)^{1} \mathbb{I} & \mathbb{I} & (-A)^{-1} \mathbb{I} & (-A)^{-2} \mathbb{I} & \cdots
\end{array}\right]
$$

where the size of the first matrix is $\infty \times n$ and the size of the second matrix is $n \times \infty$. As a consequence the rank of $R$ is at most $n$.

The $n \times n$ sub-matrix of $R$ whose upper left element is $r_{0}$ is non-singular when $(\alpha, A)$ is nonredundant [10]. As a consequence the $\alpha(-A)^{0}, \alpha(-A)^{-1}, \ldots, \alpha(-A)^{-n+1}$ row vectors as well as the $(-A)^{0} \mathbb{I},(-A)^{-1} \mathbb{I}, \ldots,(-A)^{-n+1} \mathbb{I}$ column vectors are linearly independent. 
As a consequence of Theorem 1 the determinant of any $(n+1) \times(n+1)$ submatrix of $\mathrm{R}$ must be 0 which can be exploited to define a set of equations with the (known and unknown) elements of the $r_{i}$ series as it will be discussed later.

\subsection{Defining ME distributions based on their moments}

Theorem 1 allows us to characterise the order n ME distributions based on their moments. To this end we define the following properties of moment series. Since we consider continuous distributions, $r_{0}=1$ is given.

Definition 2. Suppose we are given a $r_{i_{1}}, r_{i_{2}}, \ldots, r_{i_{k}}$ series such that $i_{1}<i_{2}<\ldots<i_{k}$ and $i_{j} \neq 0, \forall j \in$ $\{1, \ldots, k\}$. The $r_{i_{1}}, r_{i_{2}}, \ldots, r_{i_{k}}$ series is

- $A L G(n)$ feasible, if there is a vector $\alpha$ and a matrix $A$ of cardinality $n$ for which $r_{i}=\alpha(-A)^{-i} \mathbb{I}$, $\forall i \in\left\{i_{1}, \ldots, i_{k}\right\}$, but there is no such vector and matrix of cardinality $n-1$.

- $M E(n)$ feasible, if $A L G(n)$ feasible and there is a vector $\alpha$ and a matrix $A$ for which $1-\alpha e^{A t} \mathbb{I}$ is a valid cdf.

- $P H(n)$ feasible, if $M E(n)$ feasible and there is a probability vector $\alpha$ and a transient Markovian generator matrix $A$ for which $r_{i}=\alpha(-A)^{-i} \mathbb{I}, \forall i \in\left\{i_{1}, \ldots, i_{k}\right\}$.

The equivalence of the $\mathrm{ME}(2)$ and the $\mathrm{PH}(2)$ feasibilities is known and examples indicate that $\mathrm{PH}(\mathrm{n})$ is a real subset of $\mathrm{ME}(\mathrm{n})$ for $\mathrm{n}_{\imath} 2$, but the borders of these feasibility sets are hard open research problems and are out of the scope of the present paper. Due to the lack of general results on feasibilities, the statements are assigned with feasibility assumptions in this paper.

Having the ALG(n) feasible $r_{i_{1}}, r_{i_{2}}, \ldots, r_{i_{k}}$ series, where $i_{1}<i_{2}<\ldots<i_{k}, i_{k}-i_{1} \geq 2 n$ and $i_{j} \neq 0$, $\forall j \in 1, \ldots, k$

$$
\operatorname{det}\left(R_{\{0,1,2, \ldots, n\},\{i, i+1, i+2, \ldots, i+n\}}\right)=0, \quad i_{1} \leq i \leq i_{k}-2 n
$$

provides a set of $i_{k}-i_{1}-2 n+1$ equations with $i_{k}-i_{1}-k$ unknowns. These equations are not necessarily linear in the unknowns.

If $k<2 n-1$, then the number of unknowns is more than the number of equations, hence the $r_{i_{1}}, r_{i_{2}}, \ldots, r_{i_{k}}$ series can define infinitely many ME(n) distributions depending on the ME feasibility of the moments.

If $k=2 n-1$, then the number of unknowns equals to the number of equations, but at least one equation is at least quadratic in at least one unknown. This set of polynomial equations has a finite number of solutions among which there are $\operatorname{ME}(n)$ feasible and not $\operatorname{ME}(n)$ feasible series.

If $k>2 n-1$, then there are more equations than unknowns. This allows us to check the ALG(n) feasibility of the $r_{i_{1}}, r_{i_{2}}, \ldots, r_{i_{k}}$ series. If there is no solution for the set of equations the $r_{i_{1}}, r_{i_{2}}, \ldots, r_{i_{k}}$ series is not $\operatorname{ALG}(\mathrm{n})$ feasible. 


\subsection{Examples of application of (6)}

\section{Generation of the $r_{i}$ series of ME distributions}

By $R_{c_{1}, c_{2}}$ where $c_{1}$ and $c_{2}$ are two sets of indices we denote the submatrix of $R$ which consists of rows according to $c_{1}$ and columns according to $c_{2}$. For example,

$$
R_{\{1,3,4\},\{0,2\}}=\left[\begin{array}{cc}
r_{1} & r_{3} \\
r_{3} & r_{5} \\
r_{4} & r_{6}
\end{array}\right] .
$$

Theorem 1 and (6) defines the relations in the $r_{i}$ series. Based on these relations we can compose an explicit algorithm to generate any element of the $r_{i}$ series of a ME(n) distribution based on the $r_{0}, r_{1}, \ldots, r_{2 n-1}$ reduced moments without computing any representation of this distribution $(\alpha, A)$.

Given the first $2 n$ reduced moments, $r_{0}, r_{1}, \ldots, r_{2 n-1}, \operatorname{det}\left(R_{\{0,1, \ldots, n\},\{0,1, \ldots, n\}}\right)=0$ and $\operatorname{det}\left(R_{\{0,1, \ldots, n\},\{-1,0,1, \ldots, n-1\}}\right)=0$ give a single unknown linear equation for $r_{2 n}$ and $r_{-1}$, respectively. Having determined $r_{0}, \ldots, r_{2 n-1+i}, i>0,\left(r_{i}, \ldots, r_{2 n-1}, i<0\right) \operatorname{det}\left(R_{\{0,1, \ldots, n\},\{j, j+1, \ldots, j+n-1\}}\right)=0$ with $j=i+1(j=i-1)$ gives a single unknown linear equation for $r_{2 n+i}\left(r_{i-1}\right)$.

Non-unique $\mathbf{P H}(3)$ feasible solutions for $k=2 n-1$

Assuming that

$$
\begin{gathered}
r_{0}=1, r_{i_{1}}=r_{10}=0.000295999, r_{i_{2}}=r_{20}=6.998710^{-13}, r_{i_{3}}=r_{30}=1.0175810^{-23}, \\
r_{i_{4}}=r_{40}=5.2441410^{-36}, r_{i_{5}}=r_{50}=2.2301510^{-49}
\end{gathered}
$$

the set of equations in (6) is composed of $i_{k}-2 n+1=45$ equations with $i_{k}-k=45$ unknowns. There are several different solutions of this set of equations and there are more than one which are $\mathrm{PH}(3)$ feasible. E.g., the moments of

$$
\begin{aligned}
& \alpha_{1}=\left[\begin{array}{lll}
0.1 & 0.2 & 0.7
\end{array}\right] \\
& \alpha_{2}=\left[\begin{array}{lll}
0.586119 & 0.309469 & 0.104413
\end{array}\right] \\
& A_{1}=\left[\begin{array}{ccc}
-1 & 1 & 0 \\
0 & -2 & 2 \\
1 & 0 & -3
\end{array}\right] \quad \text { and } \quad A_{2}=\left[\begin{array}{ccc}
-2.51342 & 0 & 0.796945 \\
2.51342 & -2.51342 & 0 \\
0 & 0.927169 & -0.927169
\end{array}\right]
\end{aligned}
$$

are solutions of (6) for this set of reduced moments. Table 1 lists the first reduced moments of the two $\mathrm{PH}(3)$. The differences vanish for large moments, but the numerical value of the relative differences in the last row of the table $\left(\left(r_{i}^{(1)}-r_{i}^{(2)}\right) / r_{i}^{(1)}\right)$ indicates that the $r_{10}$ reduced moments are identical while the $r_{9}$ and $r_{11}$ reduced moments are different. This periodicity of the moments remain valid forever, i.e, all tenth moments of the two $\mathrm{PH}(3)$ remains identical. It is interesting to note that the probability density function (pdf) of the two $\mathrm{PH}(3)$ exhibit a similar behaviour to that of the moments (see Figure 1). The two pdfs cross each others infinitely many times. Indeed, it implies that two $\mathrm{PH}(3)$ distributions whose pdfs are identical in infinitely many points are not necessarily identical. 


\begin{tabular}{|c|cccccc|}
\hline$r_{i}$ & 0. & 1. & 2. & 3. & 4. & 5. \\
\hline$\left\{\alpha_{1}, A_{1}\right\}$ & 1. & 1.5 & 1.4875 & 1.03021 & 0.537956 & 0.224808 \\
$\left\{\alpha_{2}, A_{2}\right\}$ & 1. & 1.54536 & 1.50213 & 1.03248 & 0.538182 & 0.224824 \\
\hline$r_{i}$ & 6. & 7. & 8. & 9. & 10. & 11. \\
\hline$\left\{\alpha_{1}, A_{1}\right\}$ & 0.0782855 & 0.0233666 & 0.00610259 & 0.00141671 & 0.000295999 & 0.000056222 \\
$\left\{\alpha_{2}, A_{2}\right\}$ & 0.0782863 & 0.0233666 & 0.00610259 & 0.00141671 & 0.000295999 & 0.000056222 \\
rel. diff. & $-1.1032410^{-5}$ & $-1.5181710^{-6}$ & $-1.7830610^{-7}$ & $-1.5142210^{-8}$ & $-3.6628610^{-16}$ & $3.9414710^{-10}$ \\
\hline
\end{tabular}

Table 1: The reduced moments of $\mathrm{PH}(3)\left\{\alpha_{1}, A_{1}\right\}$ and $\left\{\alpha_{2}, A_{2}\right\}$
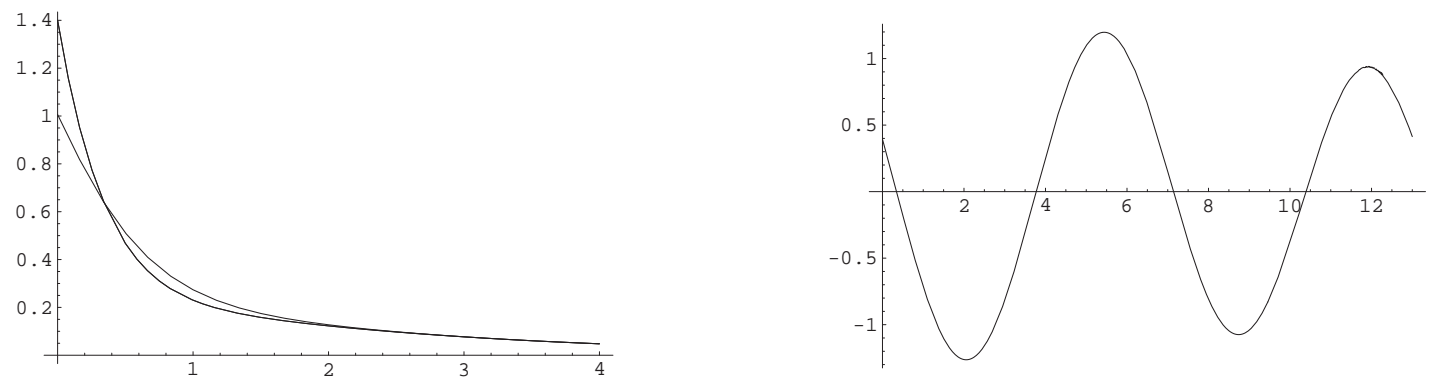

Figure 1: Density function of $\mathrm{PH}(3)\left\{\alpha_{1}, A_{1}\right\}$ and $\left\{\alpha_{2}, A_{2}\right\}$ and their difference $\left(f_{1}(t)-f_{2}(t)\right) e^{2.8 t}$

\section{Fitting 3 reduced moments out of 5}

Assume that we are given the first five reduced moments of a random variable and our aim is to construct a $\mathrm{PH}$ distribution with two phases to approximate it. The five reduced moments are $r_{1}=$ $1, r_{2}=1.25, r_{3}=4.1 \dot{6}, r_{4}=104.1 \dot{6}, r_{5}=8333 \dot{3}$. A PH distribution with two phases is determined by its first three reduced moments. However, we are not limited to use $r_{1}, r_{2}$ and $r_{3}$. For example, we can choose to fit reduced moments $r_{1}, r_{3}$ and $r_{5}$. In order to obtain PH distribution with two phases and reduced moments $r_{1}, r_{3}$ and $r_{5}$, we do the following. We assume that $r_{1}, r_{3}$ and $r_{5}$ are reduced moments of a $\mathrm{PH}$ distribution with two phases and we compute the first three reduced moments of this distribution based on (6). Then the fitting is performed based on these first three reduced moments.

In the following table we report fittings which are different in the choice of the utilised three reduced moments. In all the cases the mean is maintained and hence we have to choose two out of the four remaining reduced moments. The reduced moments that are set are indicated with bold characters. The reduced moments in the last row are chosen in such way that the sum of the relative errors in the reduced moments, $\sum_{i=2}^{5}\left|\hat{r}_{i}-r_{i}\right| / r_{i}$, are minimal. The distribution determined by this reduced moment 
set is referred to as opt.

$\begin{array}{cccc}r_{2} & r_{3} & r_{4} & r_{5} \\ \mathbf{1 . 2 5} & \mathbf{4 . 1 \dot { 6 }} & 35.5903 & 371.817 \\ \mathbf{1 . 2 5} & 6.31469 & \mathbf{1 0 4 . 1 \dot { 6 }} & 1990.25 \\ \mathbf{1 . 2 5} & 9.3729 & 265.489 & \mathbf{8 3 3 3 . \dot { 3 }} \\ 1.09182 & \mathbf{4 . 1 \dot { 6 }} & \mathbf{1 0 4 . 1 6} & 3353.48 \\ 1.05945 & \mathbf{4 . 1 \dot { 6 }} & 163.533 & \mathbf{8 3 3 3 . \mathbf { 3 }} \\ 1.01543 & 2.27679 & \mathbf{1 0 4 . 1} \dot{\mathbf{6}} & \mathbf{8 3 3 3 . \dot { 3 }} \\ 1.03826 & 3.22754 & 126.341 & 7047.53\end{array}$

In Figure 2 the pdf of the different fitted distribution are depicted. The legend indicates the reduced moments that are fitted. The figure depicts the fitting with three phases as well which matches all the five reduced moments. The logarithmic plot suggests that a better fitting of the lower moments results in a better body fitting, while the better fitting of the higher moments results better tail fitting.
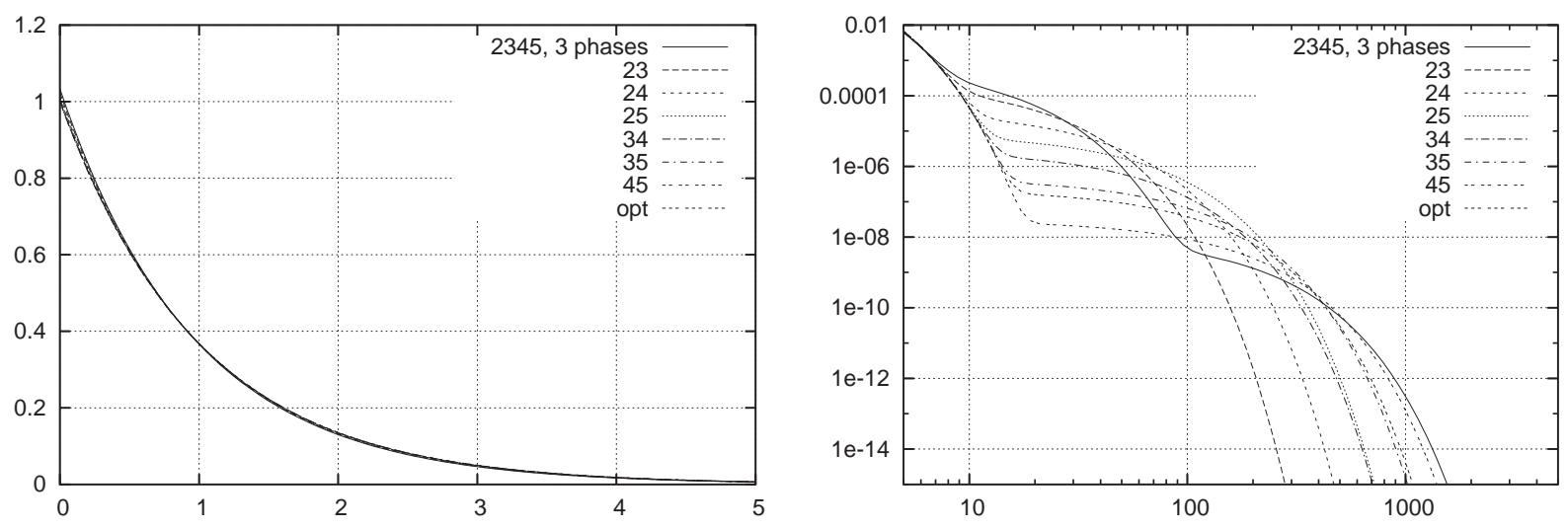

Figure 2: Body and tail of pdf of different fittings

\section{PH matching based on the derivatives of the cdf at zero}

The continuous $\mathrm{PH}(2)$ distributions are uniquely defined by their first 3 reduced moments and [15] proposes a way to compute an $\alpha$ probability vector and an $A$ generator matrix based on these reduced moments. We use this algorithm to create a "derivative matching" method for $\mathrm{PH}(2)$ distributions.

When the first three derivatives of the cdf of a $\mathrm{PH}(2)$ at $t=0$ are $r_{-1}=4, r_{-2}=17, r_{-3}=76$, we use

$$
\operatorname{det}\left[\begin{array}{ccc}
r_{-3} & r_{-2} & r_{-1} \\
r_{-2} & r_{-1} & r_{0} \\
r_{-1} & r_{0} & r_{1}
\end{array}\right]=0, \quad \operatorname{det}\left[\begin{array}{ccc}
r_{-2} & r_{-1} & r_{0} \\
r_{-1} & r_{0} & r_{1} \\
r_{0} & r_{1} & r_{2}
\end{array}\right]=0, \quad \operatorname{det}\left[\begin{array}{ccc}
r_{-1} & r_{0} & r_{1} \\
r_{0} & r_{1} & r_{2} \\
r_{1} & r_{2} & r_{3}
\end{array}\right]=0
$$

and $r_{0}=1$ to obtain $r_{1}, r_{2}, r_{3}$. Solving the equations from left to right, the number of unknown is always 1 , and the equations are linear in this unknown. The solution is

$$
r_{1}=\frac{4}{15}, \quad r_{2}=\frac{17}{225}, \quad r_{3}=\frac{76}{3375} \text {. }
$$


From $r_{1}, r_{2}, r_{3}$ we obtain $([15])$ :

$$
\alpha=\left[\begin{array}{ll}
0.2 & 0.8
\end{array}\right], \quad A=\left[\begin{array}{cc}
-3 & 3 \\
0 & -5
\end{array}\right] \text {. }
$$

\section{An example of ME(3) feasibility check based on $r_{-2}, \ldots, r_{3}$}

One can perform a numerical $\mathrm{ME}(3)$ feasibility check based on the $r_{-j}, r_{-j+1}, \ldots, r_{5-j}, 0<j<6$, series in three steps:

- compute the $r_{0}, r_{1}, \ldots, r_{5}$ series using the determinants of the $(n+1) \times(n+1)$ submatrices of $R$,

- calculate a vector $\alpha$ and a matrix $A$ based on $r_{0}, \ldots, r_{2 n-1}$ such that $r_{i}=\alpha(-A)^{-i} \mathbb{I},(i=$ $0,1, \ldots, 2 n-1)$ using the procedure of [10],

- decide the non-negativity of $f(t)=\alpha e^{A t}(-A) \mathbb{I}$.

Starting from

$$
r_{-3}=\frac{24}{5}, \quad r_{-2}=0, \quad r_{-1}=\frac{6}{5}, \quad r_{0}=1, \quad r_{1}=\frac{3}{5}, \quad r_{2}=\frac{7}{20},
$$

we can compute $r_{3}=5 / 24$ using $\operatorname{det}\left(R_{\{-3,-2,-1,0\},\{0,1,2,3\}}\right)=0, \quad r_{4}=1 / 8$ using $\operatorname{det}\left(R_{\{-2,-1,0,1\},\{0,1,2,3\}}\right)=0$ and $r_{5}=3 / 40$ using $\operatorname{det}\left(R_{\{-1,0,1,2\},\{0,1,2,3\}}\right)=0$.

Having $r_{0}, \ldots, r_{5}$ we compute $\alpha$ and $A$ which are

$$
\alpha=\left[\begin{array}{lll}
\frac{1}{3} & \frac{1}{3} & \frac{1}{3}
\end{array}\right], \quad A=\left[\begin{array}{ccc}
-\frac{152}{9} & \frac{7702}{225} & -\frac{3962}{225} \\
-\frac{395}{81} & \frac{631}{81} & -\frac{341}{81} \\
\frac{305}{81} & -\frac{721}{81} & \frac{251}{81}
\end{array}\right] .
$$

Then we plot $f(t)=\alpha e^{A t}(-A) \mathbb{I}$ as shown in Figure 3. Assuming that one can decide the non-negativity of $f(t)$ based on Figure 3 we conclude that the given $\left\{r_{-2}, r_{-1}, r_{0}, r_{1}, r_{2}, r_{3}\right\}$ series is $\operatorname{ME}(3)$ feasible.
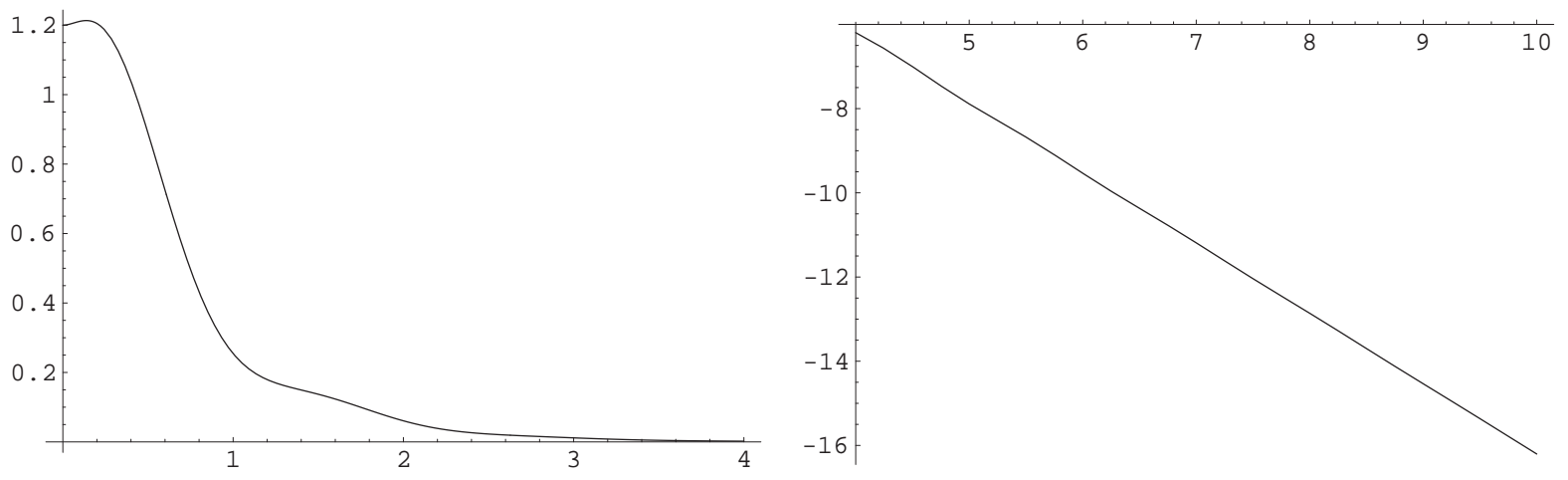

Figure 3: The density function, $f(t)$, and its tail on log-log scale constructed from the $\left\{r_{-2}, r_{-1}, r_{0}, r_{1}, r_{2}, r_{3}\right\}$ series 


\section{Matrix Exponential and Markov Arrival Processes}

\subsection{Preliminaries}

Let $X(t)$ be a stationary arrival process, defined by matrices $A_{0}$ and $A_{1}$, whose sequence of interarrival times is $X_{0}, X_{1}, \ldots$ The joint density of $X_{0}, X_{1}, \ldots, X_{m}$ is

$$
f\left(x_{0}, x_{1}, \ldots, x_{m}\right)=\alpha e^{A_{0} x_{0}} A_{1} e^{A_{0} x_{1}} A_{1} \ldots e^{A_{0} x_{m}} A_{1} \mathbb{I}
$$

where $\alpha$ is the solution of $\alpha\left(-A_{0}\right)^{-1} A_{1}=\alpha$ and $\alpha \mathbb{I}=1$. Here again, without loss of generality [14] we assume that the closing vector is $\mathbb{I}$. The marginal densities of $f\left(x_{0}, x_{1}, \ldots, x_{m}\right)$ can be obtained using $\int_{0}^{\infty} e^{A_{0} x} d x=\left(-A_{0}\right)^{-1}$. For example, $f\left(x_{0}, x_{2}, x_{3}, \ldots, x_{m}\right)=$ $\alpha e^{A_{0} x_{0}} A_{1}\left(-A_{0}\right)^{-1} A_{1} e^{A_{0} x_{2}} A_{1} \ldots e^{A_{0} x_{m}} A_{1} \mathbb{I}$.

The cardinality of the square matrices $A_{0}$ and $A_{1}$ is $n$. Similarly to the previous section, we consider the following cases:

- If $f\left(x_{0}, x_{1}, \ldots, x_{m}\right) \geq 0, \quad \forall \quad m \geq 0$ and $\forall \quad x_{1}, x_{2}, \ldots, x_{m} \geq 0$ and $\int_{x_{1}} \ldots \int_{x_{m}} f\left(x_{0}, x_{1}, \ldots, x_{m}\right) d x_{1} \ldots d x_{m}=1, \forall m \geq 0$, then $X(t)$ is a matrix-exponential process of order $n, \operatorname{MEP}(\mathrm{n})$. MEP is identical to the rational arrival process defined in [4].

- If $A_{0}$ is a transient Markovian generator matrix and $A_{1} \geq 0$, then $X(t)$ is a Markov arrival process of order $n, \operatorname{MAP}(\mathrm{n})$.

When $X(t)$ is a $\operatorname{MEP}(\mathrm{n})$, it has the following properties:

- The stationary inter-arrival time distribution is matrix-exponential with parameters $\alpha$ and $A_{0}$. Therefore, $A_{0}$ fulfills the conditions of ME distributions provided in the previous section.

- Matrix $G=\left(-A_{0}\right)^{-1} A_{1}$ governs the evolution of the initial vectors $\left(\alpha_{1}, \alpha_{2}, \ldots\right)$ starting from an arbitrary initial vector $\left(\alpha_{0}\right)$.

- Assuming that the initial vector is $\alpha_{0}$ the marginal distribution $f\left(x_{1}, x_{2}, x_{3}, \ldots, x_{m}\right)$ gives $\alpha_{1}=\alpha_{0} G$ and consequently $\alpha_{i}=\alpha_{i-1} G$.

- From the marginal distribution $f\left(x_{0}, x_{1}, x_{2}, \ldots, x_{m-1}\right)$ we have $G \mathbb{I}=\mathbb{I}$.

$-\mathbb{I}=G \mathbb{I}=\left(-A_{0}\right)^{-1} A_{1} \mathbb{I}$ implies $-A_{0} \mathbb{I}=A_{1} \mathbb{I}$.

- If $\alpha_{0} \mathbb{I}=1$, then $\mathbb{I}=G \mathbb{I}$ also implies that $\alpha_{i} \mathbb{I}=\alpha_{0} G^{i} \mathbb{I}=1$.

- If starting from any arbitrary $\alpha_{0}$ (satisfying $\alpha_{0} \mathbb{I}=1$ ) the initial vectors $\left(\alpha_{1}, \alpha_{2}, \ldots\right)$ converge to $\alpha$, then matrix $G$ has $n$ eigenvalues on the unit disk and one of them is 1 (otherwise if matrix $G$ has an eigenvalue out of the unit disk then the $\alpha_{1}, \alpha_{2}, \ldots$ series diverge and if all eigenvalues are on the unit disk, but two of them equal to 1 then $\lim _{i \rightarrow \infty} \alpha_{i}$ depends on $\alpha_{0}$ ).

When $X(t)$ is a $\operatorname{MAP}(\mathrm{n})$, it has the following additional properties:

- The phases of the system at arrival epochs form a DTMC, which means that matrix $G$ is a transition probability matrix, or stochastic matrix, i.e., the elements of $G$ are non-negative and the rows of $G$ sum up to 1 . 
- $\alpha$ is a probability vector.

The major differences of the MEP and the MAP cases are the following. In the case of MEPs the row sum and the diagonal element of $A_{0}$ can be positive, the elements of $\alpha$ and $G$ can be negative or greater than one and $A_{1}$ can contain negative elements. However, the row sums of $A_{0}+A_{1}$ must be zero in both cases.

The pair of square matrices $\left(A_{0}, A_{1}\right)$ is referred to the representation of the MEP (MAP). In general, the $\left(A_{0}, A_{1}\right)$ representation is not unique.

Definition 3. An $\left(A_{0}, A_{1}\right)$ representation of cardinality $n$ is said to be non-redundant if the inter-arrival time distribution defined by $\left(A_{0}, A_{1}\right)$ is a non redundant $M E(n)$ distribution.

Throughout the paper we assume that the representations of MEPs (MAPs) are non-redundant.

Since the inter-arrival times have a $\mathrm{ME}(\mathrm{n})$ distribution with generator $A_{0}$ and initial vector $\alpha$, the reduced moments of the inter-arrival times are (in accordance with (4)) $r_{i}=\alpha\left(-A_{0}\right)^{-i} \mathbb{I}$, and the results of the previous section are applicable to compute the relation of the elements of the $r_{i}$ series for $i=\{0, \pm 1, \pm 2, \ldots\}$.

The joint moment of the $X_{0}, X_{a_{1}}, \ldots, X_{a_{m-1}}$ inter-arrival times, where $a_{0}=0<a_{1}<a_{2}<\ldots<$ $a_{m-1}$, can be computed as

$$
E\left(X_{0}^{i_{1}} X_{a_{1}}^{i_{2}} \ldots X_{a_{m-1}}^{i_{m}}\right)=\alpha i_{1} !\left(-A_{0}\right)^{-i_{1}} G^{a_{1}-a_{0}} i_{2} !\left(-A_{0}\right)^{-i_{2}} \ldots G^{a_{m-1}-a_{m-2}} i_{m} !\left(-A_{0}\right)^{-i_{m}} \mathbb{I}
$$

To shorten the notation we introduce $E=\left(-A_{0}\right)^{-1}$ and

$$
\gamma_{k_{2}, \ldots, k_{m}}^{i_{1}, i_{2}, \ldots, i_{m}}=\frac{1}{\prod_{j=1}^{m} i_{j} !} E\left(X_{0}^{i_{1}} X_{k_{2}}^{i_{2}} \ldots X_{k_{2}+\ldots+k_{m}}^{i_{m}}\right)=\alpha E^{i_{1}} G^{k_{2}} E^{i_{2}} G^{k_{3}} E^{i_{3}} \ldots G^{k_{m}} E^{i_{m}} \mathbb{I} .
$$

We refer to the $\gamma_{k_{2}, \ldots, k_{m}}^{i_{1}, i_{2}, \ldots, i_{m}}$ as reduced joint moments. Special cases of (8) include

- $m=1, i_{1}=i$ :

$$
E\left(X_{0}^{i}\right)=i ! \alpha\left(-A_{0}\right)^{-i} \mathbb{I}=\gamma^{i}=r_{i},
$$

indicates that $\gamma_{k_{2}, \ldots, k_{m}}^{i_{1}, i_{2}, \ldots, i_{m}}$ is a generalisation of the reduced moment series. Since $\gamma^{i}=r_{i}$, we use the $\gamma_{i}$ notation to emphasise the relation with other $\gamma_{k_{2}, \ldots, k_{m}}^{i_{1}, i_{2}, \ldots, i_{m}}$ quantities and the $r_{i}$ notation to refer to the results of Section 2 in the remainder of the paper.

- $m=2, a_{1}=k, i_{1}=i_{2}=1$ : The joint mean,

$$
E\left(X_{0} X_{k}\right)=\alpha\left(-A_{0}\right)^{-1} G^{k}\left(-A_{0}\right)^{-1} \mathbb{I}=\gamma_{k}^{1,1}
$$

is the basic quantity to characterise the lag-k correlation of the process.

- $m=2, a_{1}=k$ : The joint moments of the inter-arrival times $X_{0}$ and $X_{k}$,

$$
E\left(X_{0}^{i_{1}} X_{k}^{i_{2}}\right)=i_{1} ! i_{2} ! \alpha\left(-A_{0}\right)^{-i_{1}} G^{k}\left(-A_{0}\right)^{-i_{2}} \mathbb{I}=i_{1} ! i_{2} ! \gamma_{k}^{i_{1}, i_{2}}
$$

carries information about the joint distribution of the k-apart inter-arrival times. We refer to $E\left(X_{0}^{i} X_{k}^{j}\right)$ as lag- $k$ quantities. 
The following subsections extend the concept of a Hankel matrix to find the relation between the various moments of the inter-arrival times of $\operatorname{MEP}(n)$ s. Similar to the ME distribution case, we present methods to compute the relations between all possible reduced moments of the inter-arrival times without computing any $\left(A_{0}, A_{1}\right)$ representation. We show that the first $2 n-1$ reduced moments of the inter-arrival times, $E\left(X_{0}^{i}\right), 1 \leq i \leq 2 n-1$, and the first $(n-1)^{2}$ lag-1 reduced moments, $E\left(X_{0}^{i} X_{1}^{j}\right), 1 \leq i, j \leq n-1$, uniquely determine all other reduced moments of a non-redundant $\operatorname{MEP}(\mathrm{n})$, and we present an algorithm for computing them.

\subsection{Relation of reduced joint moments of a $\operatorname{MEP}(n)$ processes}

Let

$$
M_{1}\left(i_{1}, \ldots, i_{m}, k_{2}, \ldots, k_{m}\right)=\left[\begin{array}{ccccc}
\gamma_{k_{2}, \ldots, i_{m}}^{i_{1}, i_{2}, \ldots, i_{m}} & \gamma_{k_{2}, \ldots, k_{m-1}}^{i_{1}, i_{2}, \ldots, i_{m-1}} & \gamma_{k_{2}, \ldots, k_{m-1}}^{i_{1}, i_{2}, \ldots, i_{m-1}+1} & \ldots & \gamma_{k_{2}, \ldots, k_{m-1}}^{i_{1}, i_{2}, \ldots, i_{m-1}+n-1} \\
\gamma^{i_{m}} & r_{0} & r_{1} & \ldots & r_{n-1} \\
\gamma_{k_{m}}^{1, i_{m}} & r_{1} & r_{2} & \ldots & r_{n} \\
\gamma_{k_{m}}^{2, i_{m}} & r_{2} & r_{3} & \ldots & r_{n+1} \\
\vdots & \vdots & \vdots & \vdots & \vdots \\
\gamma_{k_{m}}^{n-1, i_{m}} & r_{n-1} & r_{n} & \ldots & r_{2 n-2}
\end{array}\right]
$$

and

$$
M_{2}\left(i_{1}, i_{2}, k\right)=\left[\begin{array}{ccccc}
\gamma_{k}^{i_{1}, i_{2}} & \gamma^{i_{1}} & \gamma_{1}^{i_{1}, 1} & \ldots & \gamma_{1}^{i_{1}, n-1} \\
\gamma^{i_{2}} & r_{0} & r_{1} & \ldots & r_{n-1} \\
\gamma_{k-1}^{1, i_{2}} & r_{1} & r_{2} & \ldots & r_{n} \\
\gamma_{k-1}^{2, i_{2}} & r_{2} & r_{3} & \ldots & r_{n+1} \\
\vdots & \vdots & \vdots & \vdots & \vdots \\
\gamma_{k-1}^{n-1, i_{2}} & r_{n-1} & r_{n} & \ldots & r_{2 n-2}
\end{array}\right] .
$$

Theorem 2. Under the non-redundant condition the rank of $M_{1}$ and $M_{2}$ is $n$.

Proof.

$$
\begin{aligned}
& M_{1}\left(i_{1}, \ldots, i_{m}, k_{2}, \ldots, k_{m}\right)= \\
& {\left[\begin{array}{c}
\alpha E^{i_{1}} G^{k_{2}} E^{i_{2}} \ldots G^{k_{m-1}} E^{i_{m-1}} \\
\alpha \\
\alpha E \\
\alpha E^{2} \\
\vdots \\
\alpha E^{n-1}
\end{array}\right]\left[\begin{array}{lllllll}
G^{k_{m}} E^{i_{m}} \mathbb{I} & \mathbb{I} & E^{1} \mathbb{I} & E^{2} \mathbb{I} & \ldots & E^{n-1} \mathbb{I}
\end{array}\right],}
\end{aligned}
$$




$$
M_{2}\left(i_{1}, i_{2}, k\right)=\left[\begin{array}{c}
\alpha E^{i_{1}} G \\
\alpha \\
\alpha E \\
\alpha E^{2} \\
\vdots \\
\alpha E^{n-1}
\end{array}\right]\left[\begin{array}{llllll}
G^{k-1} E^{i_{2}} \mathbb{I} & \mathbb{I} & E^{1} \mathbb{I} & E^{2} \mathbb{I} & \ldots & E^{n-1} \mathbb{I}
\end{array}\right]
$$

and the rest of the proof is the same as for Theorem 1.

From Theorem 2, the equation

$$
\operatorname{det}\left(M_{\ell}\left(i_{1}, \ldots, i_{m}, k_{2}, \ldots, k_{m}\right)\right)=0, \quad \ell=1,2
$$

establishes the basic relation of the reduced joint moments of MEP(n)s. The upper left element of $M_{1}\left(i_{1}, \ldots, i_{m}, k_{2}, \ldots, k_{m}\right)$ and $M_{2}\left(i_{1}, i_{2}, k\right)$ are the ones with the highest order and (10) gives an explicit expression to compute these elements from the lower order reduced joint moments of the same process.

\subsection{Computation of any reduced joint moment of $\operatorname{MEP}(n)$ processes}

Assume that the parameters, $r_{0}=1, r_{i}=\gamma^{i}$ for $1 \leq i \leq 2 n-1$ and $\gamma_{1}^{i, j}$ for $1 \leq i, j \leq n-1$ are known. Our aim is to compute $\gamma_{k_{2}, \ldots, k_{m}}^{i_{1}, i_{2}, \ldots, i_{m}}$ with $m>1$. This can be done by the iterative application of (10).

Based on $M_{1}\left(i_{1}, \ldots, i_{m}, k_{2}, \ldots, k_{m}\right)$, it is possible to obtain an explicit expression for $\gamma_{k_{2}, \ldots, k_{m}}^{i_{1}, i_{2}, \ldots, i_{m}}$ in terms of

- $\gamma_{k_{2}, \ldots, k_{m-1}}^{i_{1}, i_{2}, \ldots, i_{m-1}+j}, 0 \leq j \leq n-1$,

- $\gamma_{k_{m}}^{j, i_{m}}, 1 \leq j \leq n-1$,

- $\gamma^{j}, 1 \leq j$.

Then by constructing the matrices $M_{1}\left(i_{1}, \ldots, i_{m-1}+j, k_{2}, \ldots, k_{m-1}\right), 0 \leq j \leq n-1$ we can obtain explicit expressions for the quantities $\gamma_{k_{2}, \ldots, k_{m-1}}^{i_{1}, i_{2}, \ldots, i_{m-1}+j}, 0 \leq j \leq n-1$ in terms of

- $\gamma_{k_{2}, \ldots, k_{m-2}}^{i_{1}, i_{2}, \ldots, i_{m-2}+j}, 0 \leq j \leq n-1$,

- $\gamma_{k_{m}}^{j_{1}, i_{m-1}+j_{2}}, 1 \leq j_{1} \leq n-1,0 \leq j_{2} \leq n-1$,

- $\gamma^{j}, 1 \leq j$.

By repeating the above step finally we obtain an expression for $\gamma_{k_{2}, \ldots, k_{m}}^{i_{1}, i_{2}, \ldots, i_{m}}$ in terms of quantities such as

- $\gamma_{k}^{j, i}, 1 \leq j \leq n-1$,

- $\gamma^{j}, 1 \leq j$.

Quantities such as $\gamma^{j}=r_{j}, 1 \leq j$ can be computed as described in Section 2. $M_{2}\left(i_{1}, i_{2}, k\right)$ will be used instead to deal with the quantities such as $\gamma_{k}^{j, i}, 1 \leq j \leq n-1$. Based on (10), it is possible the construct an explicit expression for $\gamma_{k}^{i_{1}, i_{2}}$ in terms of quantities such as

- $\gamma_{1}^{i_{1}, j}, 1 \leq j \leq n-1$, 
- $\gamma_{k-1}^{j, i_{2}}, 1 \leq j \leq n-1$,

- $\gamma^{j}, 1 \leq j$.

Since $1 \leq i_{1} \leq n-1$, the quantities $\gamma_{1}^{i_{1}, j}, 1 \leq j \leq n-1$ are assumed to be known, while $\gamma^{j}, 1 \leq j$ can be computed as described in Section 2. In order to deal with $\gamma_{k-1}^{j, i_{2}}, 0 \leq j \leq n-1$ we construct $M_{2}\left(j, i_{2}, k-1\right), 1 \leq j \leq n-1$ from which we can have expressions for these quantities in term of

- $\gamma_{1}^{j_{1}, j_{2}}, 1 \leq j_{1}, j_{2} \leq n-1$,

- $\gamma_{k-2}^{j, i_{2}}, 1 \leq j \leq n-1$,

- $\gamma^{j}, 1 \leq j$.

By successive application of the above step we obtain an expression for $\gamma_{k}^{j, i}, 1 \leq j \leq n-1$ in terms of

- $\gamma_{1}^{j, i}, 1 \leq j \leq n-1$,

- $\gamma^{j}, 1 \leq j$.

The quantities $\gamma_{1}^{j, i}, 1 \leq j \leq n-1$ can instead be computed by constructing the following special case of the $M_{2}\left(i_{1}, i_{2}, k\right)$ matrix.

$$
\begin{gathered}
M_{2}\left(i_{1}, i_{2}, 1\right)= \\
{\left[\begin{array}{c}
\alpha E^{i_{1}} G \\
\alpha \\
\alpha E \\
\alpha E^{2} \\
\vdots \\
\alpha E^{n-1}
\end{array}\right]\left[\begin{array}{llllll}
E^{i_{2}} \mathbb{I} & \mathbb{I} & E^{1} \mathbb{I} & E^{2} \mathbb{I} & \ldots & E^{n-1} \mathbb{I}
\end{array}\right]=\left[\begin{array}{ccccc}
\gamma_{1}^{i_{1}, i_{2}} & \gamma^{i_{1}} & \gamma_{1}^{i_{1}, 1} & \ldots & \gamma_{1}^{i_{1}, n-1} \\
\gamma^{i_{2}} & r_{0} & r_{1} & \ldots & r_{n-1} \\
\gamma^{i_{2}+1} & r_{1} & r_{2} & \ldots & r_{n} \\
\gamma^{i_{2}+2} & r_{2} & r_{3} & \ldots & r_{n+1} \\
\vdots & \vdots & \vdots & \vdots & \vdots \\
\gamma^{i_{2}+n-1} & r_{n-1} & r_{n} & \ldots & r_{2 n-2}
\end{array}\right] .}
\end{gathered}
$$

Based on (11), it is possible to construct an explicit expression for $\gamma_{1}^{i_{1}, i_{2}}$ in terms of quantities that are all known since $1 \leq i_{1} \leq n-1$ or can be computed by the algorithm given in Section 2 and $\gamma_{1}^{i_{1}, i_{2}}$ is given for $1 \leq i_{1}, i_{2} \leq n-1$.

\section{Application of the procedure for computing $E\left(X_{0}^{2} X_{2} X_{3}\right)$}

In the following we list the matrices that we need to compute $\gamma_{2,1}^{2,1,1}$ from which it is straightforward to determine $E\left(X_{0}^{2} X_{2} X_{3}\right)$ as a function of the known parameters. We assume that $n=2$ and hence the matrices are of size $3 \times 3$ and we know $\gamma^{i}, 0 \leq i \leq 3$ and $\gamma_{1}^{1,1}$. All the matrices are used to obtain an expression for the $(1,1)$ entry of the matrix based on the fact that the determinant of the matrix must be 0 . In all the matrices bold characters are used to indicate the variables that we do not know yet.

$$
M_{1}(2,1,1,2,1)=\left[\begin{array}{ccc}
\gamma_{2,1}^{2,1,1} & \gamma_{\mathbf{2}}^{\mathbf{2 , 1}} & \gamma_{\mathbf{2}}^{\mathbf{2 , 2}} \\
\gamma^{1} & \gamma^{0} & \gamma^{1} \\
\gamma_{1}^{1,1} & \gamma^{1} & \gamma^{2}
\end{array}\right], M_{1}(2,1,2)=\left[\begin{array}{ccc}
\gamma_{2}^{2,1} & \gamma^{2} & \gamma^{3} \\
\gamma^{1} & \gamma^{0} & \gamma^{1} \\
\gamma_{\mathbf{2}}^{\mathbf{1}, \mathbf{1}} & \gamma^{1} & \gamma^{2}
\end{array}\right], M_{1}(2,2,2)=\left[\begin{array}{ccc}
\gamma_{2}^{2,2} & \gamma^{2} & \gamma^{3} \\
\gamma^{2} & \gamma^{0} & \gamma^{1} \\
\gamma_{\mathbf{2}}^{\mathbf{1}, 2} & \gamma^{1} & \gamma^{2}
\end{array}\right],
$$




$$
M_{2}(1,1,2)=\left[\begin{array}{ccc}
\gamma_{2}^{1,1} & \gamma^{1} & \gamma_{1}^{1,1} \\
\gamma^{1} & \gamma^{0} & \gamma^{1} \\
\gamma_{1}^{1,1} & \gamma^{1} & \gamma^{2}
\end{array}\right], M_{2}(1,2,2)=\left[\begin{array}{ccc}
\gamma_{2}^{1,2} & \gamma^{1} & \gamma_{1}^{1,1} \\
\gamma^{2} & \gamma^{0} & \gamma^{1} \\
\gamma_{1}^{1,2} & \gamma^{1} & \gamma^{2}
\end{array}\right], M_{2}(1,2,1)=\left[\begin{array}{ccc}
\gamma_{1}^{1,2} & \gamma^{1} & \gamma_{1}^{1,1} \\
\gamma^{2} & \gamma^{0} & \gamma^{1} \\
\gamma^{3} & \gamma^{1} & \gamma^{2}
\end{array}\right]
$$

Based on the above matrices and the facts that $\gamma^{0}=1$ and $\gamma^{i}=r_{i}$ we have that

$$
\begin{aligned}
\gamma_{2,1}^{2,1,1}= & \frac{1}{\left(\left(r_{1}\right)^{2}-r_{2}\right)^{4}}\left(r _ { 2 } \left(\gamma_{1}^{1,1}\left(r_{2}\right)^{4}-\left(r_{1}\right)^{4} \gamma_{1}^{1,1}\left(\left(\gamma_{1}^{1,1}\right)^{2}+4 \gamma_{1}^{1,1} r_{2}-8\left(r_{2}\right)^{2}\right)+\right.\right. \\
& \left.\left(r_{1}\right)^{2} \gamma_{1}^{1,1} r_{2}\left(2\left(\gamma_{1}^{1,1}\right)^{2}-\gamma_{1}^{1,1} r_{2}-4\left(r_{2}\right)^{2}\right)+\left(r_{1}\right)^{6}\left(2\left(\gamma_{1}^{1,1}\right)^{2}-2 \gamma_{1}^{1,1} r_{2}-\left(r_{2}\right)^{2}\right)\right)+ \\
& \left.r_{1}\left(\left(r_{1}\right)^{2}-\gamma_{1}^{1,1}\right)^{2}\left(r_{2}\left(-3 \gamma_{1}^{1,1}+r_{2}\right)+\left(r_{1}\right)^{2}\left(\gamma_{1}^{1,1}+r_{2}\right)\right) r_{3}-\left(\left(r_{1}\right)^{2}-\gamma_{1}^{1,1}\right)^{3}\left(r_{3}\right)^{2}\right) .
\end{aligned}
$$

\subsection{Defining $\operatorname{MEP}(\mathrm{n})$ based on their reduced joint moments}

We have seen that the $r_{0}=1, r_{i}, 1 \leq i \leq 2 n-1$ reduced moments and the $\gamma_{1}^{i, j}, 1 \leq i, j \leq n-1$ reduced joint moments uniquely defines any reduced joint moment of a $\operatorname{MEP}(n)$. By this reason we define the basic moment set as follows.

Definition 4. The basic moment set of a $\operatorname{MEP}(n)$ is the set of $r_{0}=1, r_{i}, 1 \leq i \leq 2 n-1$ reduced moments together with the $\gamma_{1}^{i, j}, 1 \leq i, j \leq n-1$ reduced joint moments.

The basic moment set is composed of $n^{2}$ moments and an additive constraint, $r_{0}=1$.

Similar to Subsection 2.3, (10) provides a set of equations to determine the basic moment set of a $\operatorname{MEP}(\mathrm{n})$ based on its arbitrary reduced joint moments. To investigate this set of equations we extend the definitions of feasibility.

Definition 5. Suppose we are given a $\gamma_{\underline{k}(\ell)}^{i(\ell)}, 1 \leq \ell \leq L$, series where $\underline{i}(\ell)$ and $\underline{k}(\ell)$ denote series of $i_{1}, i_{2}, \ldots, i_{m}$ and $k_{2}, \ldots, k_{m}$ indexes, respectively. The $\gamma_{\underline{\underline{k}}(\ell)}^{\underline{i(\ell)}}, 1 \leq \ell \leq L$, series is

- ALG2(n) feasible, if there is a pair of square matrixes $A_{0}$ and $A_{1}$ of cardinality $n$ for which (9) results in the given $\gamma_{\underline{\underline{k}}(\ell)}^{(\ell)}, 1 \leq \ell \leq L$, series, but there are no such pair of matrixes of cardinality $n-1$.

- $M E P(n)$ feasible, if $A L G 2(n)$ feasible and there is a pair of square matrixes $A_{0}$ and $A_{1}$ for which all joint densities, defined by (7), are non-negative.

- $M A P(n)$ feasible, if $\operatorname{MEP}(n)$ feasible and there is a pair of square matrices $A_{0}$ and $A_{1}$, for which $A_{0}$ is a transient Markovian generator matrix and $A_{1}$ is non-negative.

The equivalence of the $\operatorname{MAP}(2)$ and the $\operatorname{MEP}(2)$ feasibilities is presented in [16] and, similar to the relation of the $\mathrm{PH}(\mathrm{n})$ and the $\mathrm{ME}(\mathrm{n})$ feasibility sets, examples indicate that $\mathrm{MAP}(\mathrm{n})$ is a real subset of $\operatorname{MEP}(n)$ for $n_{i} 2$. The borders of the $\operatorname{MAP}(n)$ and the $\operatorname{MEP}(n)$ feasibility sets are still open research problems and are out of the scope of the present paper.

Let $\Gamma\left(\gamma_{\underline{k}}^{\underline{\underline{i}}(\ell)}\right)$ be the set of equations that defines the relation of the $\gamma_{\underline{\underline{k}}(\ell)}^{\underline{i(\ell)}}$ reduced joint moment with the elements of the basic moment set as it is provided in the previous subsection. 


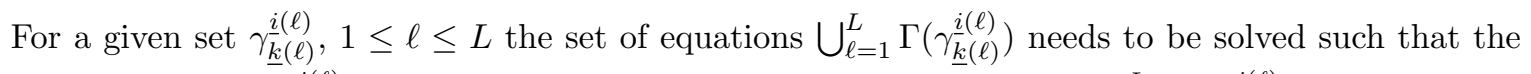
known quantities are $\gamma_{\underline{k}}^{(\ell(\ell)}, 1 \leq \ell \leq L$ and all other quantities that appear in $\bigcup_{\ell=1}^{L} \Gamma\left(\gamma_{\underline{k}}^{(\ell(\ell)}\right)$ are unknowns. Usually, the equations of $\bigcup_{\ell=1}^{L} \Gamma\left(\gamma_{\underline{k}}^{\underline{i}(\ell)}\right)$ are nonlinear in the unknowns.

We can distinguish main 3 cases. The set of equations $\bigcup_{\ell=1}^{L} \Gamma\left(\gamma_{\underline{k}}^{\underline{i}(\ell)}\right)$ has

- infinite solutions - there are less than $n^{2}$ independent equations,

- a finite number of solutions,

- no solutions - the moment set $\gamma_{\underline{k}(\ell)}^{i(\ell)}, 1 \leq \ell \leq L$ is not ALG2(n) feasible.

Unfortunately, it is not as easy to recognise the independent equations in $\bigcup_{\ell=1}^{L} \Gamma\left(\gamma_{\underline{k}(\ell)}^{\underline{i}(\ell)}\right)$ as it was in case of ME distributions. In the following two subsections two examples demonstrate this difficulty.

\subsection{Dependent $\operatorname{MEP}(\mathrm{n})$ moments}

Suppose we are given the $r_{1}, r_{2}, r_{3}, r_{4}, r_{5}$ reduced moments and the $\gamma_{1}^{1,1}, \gamma_{1}^{1,2}, \gamma_{1}^{1,3}, \gamma_{1}^{1,4}$ reduced joint moments of a $\operatorname{MEP}(3)$. Similar to the number of moments in the basic moment set, this is a set of $n^{2}$ moments and based on this fact one might expect to compute all other moments of the MEP(n) from this moment set.

We have that $\operatorname{det}\left(M_{2}(1,3,1)\right)=0$ and $\operatorname{det}\left(M_{2}(1,4,1)\right)=0$. These equations define relations for the given set of moments, that is,

$$
M_{2}(1,3,1)=\left[\begin{array}{cccc}
\gamma_{1}^{1,3} & \gamma^{1} & \gamma_{1}^{1,1} & \gamma_{1}^{1,2} \\
\gamma^{3} & \gamma^{0} & \gamma^{1} & \gamma^{2} \\
\gamma^{4} & \gamma^{1} & \gamma^{2} & \gamma^{3} \\
\gamma^{5} & \gamma^{2} & \gamma^{3} & \gamma^{4}
\end{array}\right], M_{2}(1,4,1)=\left[\begin{array}{cccc}
\gamma_{1}^{1,4} & \gamma^{1} & \gamma_{1}^{1,1} & \gamma_{1}^{1,2} \\
\gamma^{4} & \gamma^{0} & \gamma^{1} & \gamma^{2} \\
\gamma^{5} & \gamma^{1} & \gamma^{2} & \gamma^{3} \\
\gamma^{6} & \gamma^{2} & \gamma^{3} & \gamma^{4}
\end{array}\right] .
$$

It can be seen that $\operatorname{det}\left(M_{2}(1,3,1)\right)=0$ and $\operatorname{det}\left(M_{2}(1,4,1)\right)=0$ can be used to determine $\gamma_{1}^{1,3}, \gamma_{1}^{1,4}$, as a function of $r_{1}, r_{2}, r_{3}, r_{4}, r_{5}$ and $\gamma_{1}^{1,1}, \gamma_{1}^{1,2}$, using that $r_{0}, r_{1}, r_{2}, r_{3}, r_{4}, r_{5}$ also defines $r_{6}=\gamma^{6}$.

The $\operatorname{det}\left(M_{2}(1,3,1)\right)=0$ equation can also be used to show that the $r_{1}, r_{2}, r_{3}, r_{4}$ reduced moments and the $\gamma_{1}^{1,1}, \gamma_{1}^{1,2}, \gamma_{1}^{1,3}, \gamma_{1}^{2,1}, \gamma_{1}^{2,2}$ reduced joint moments uniquely determine the elements of the basic moment set.

From this example we draw the following conclusions. It is not obvious to see the dependencies of the various sets of moments. There is more than one set of $n^{2}$ moments that uniquely determine all moments of a $\operatorname{MEP}(n)$ process and, therefore, the choice of the basic moment set is not unique.

\subsection{Two different MEPs with equal marginal distribution and lag- correlations}

In [13] a procedure is presented for the construction of a MEP based on the first $2 n-1$ moments of the marginal distribution and the first $2 n-3$ lag-correlations. Here we show that there are different MEPs that realize the same marginal distribution and lag-correlations. 
Assume that $n=3$ and we are given the basic moment set of the MEP(3), in particular we have $\gamma_{1}^{1,2}=x_{1}$ and $\gamma_{1}^{2,1}=x_{2}$. Based on these parameters, as shown in Subsection 3.3, it is possible to compute the quantities that determine the lag-correlations. By following these computations, it can be seen that the given parameter set with $\gamma_{1}^{1,2}=x_{1}$ and $\gamma_{1}^{2,1}=x_{2}$ results in the same lag-correlations as the ones with $\gamma_{1}^{1,2}=x_{2}$ and $\gamma_{1}^{2,1}=x_{1}$.

As an example we consider the following two cases:

$$
\begin{gathered}
r_{1}=\frac{559}{1350}, r_{2}=\frac{469081}{1215000}, r_{3}=\frac{4660039019}{12028500000}, r_{4}=\frac{4237895351171}{10825650000000}, r_{5}=\frac{42422816639765929}{107173935000000000}, \\
E\left(X_{0}^{1} X_{1}^{1}\right)=\frac{1309691}{26730000}, E\left(X_{0}^{2} X_{1}^{2}\right)=\frac{525968309171}{5412825000000}, \\
E\left(X_{0}^{1} X_{1}^{2}\right)=\frac{703719119}{12028500000} \text { or } \frac{1031769119}{12028500000}, \\
E\left(X_{0}^{2} X_{1}^{1}\right)=\frac{1031769119}{12028500000} \text { or } \frac{703719119}{12028500000} .
\end{gathered}
$$

In both cases the lag-correlations are identical and the first lag-correlations are

$$
E\left(X_{0} X_{2}\right)=\frac{4146491}{17820000}, \quad E\left(X_{0} X_{3}\right)=\frac{3011771}{21384000}, \quad E\left(X_{0} X_{4}\right)=\frac{39937801}{213840000}, \quad E\left(X_{0} X_{5}\right)=\frac{23351837}{142560000} .
$$

In order to show that the two MEPs are different, in Figure 4 we depict the joint density of $X_{0}$ and $X_{1}$ for a given value of $X_{0}$.

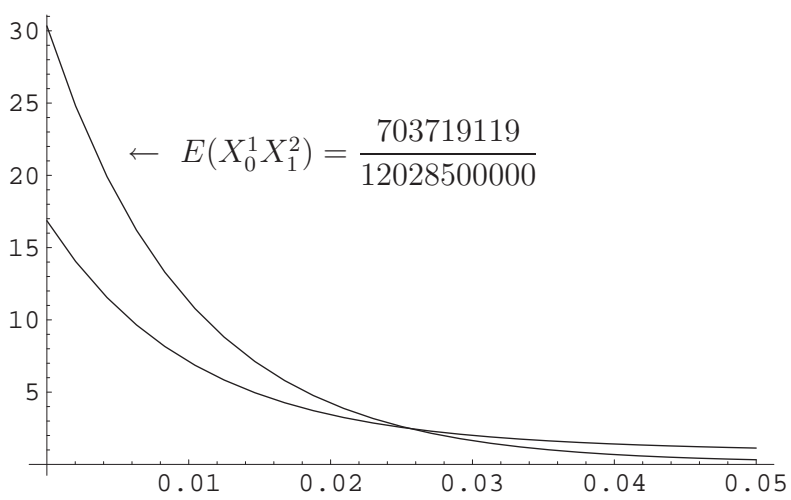

Figure 4: Joint density, $f_{X_{0}, X_{1}}(1, x)$, for the two MEPs with equal marginal moments and lag-correlation

\subsection{Extension to negative indexes}

Similar to the reduced moments of ME distributions, we can extend the definition of $\gamma_{k_{2}, \ldots, k_{m}}^{i_{1}, i_{2}, \ldots, i_{m}},(9)$, for negative $i_{j}$ indices such that

$$
\gamma_{k_{2}, \ldots, k_{m}}^{i_{1}, i_{2}, \ldots, i_{m}}=\alpha E^{i_{1}} G^{k_{2}} E^{i_{2}} G^{k_{3}} E^{i_{3}} \ldots G^{k_{m}} E^{i_{m}} \mathbb{I}
$$

The probabilistic interpretation of these quantities is less evident, but there are exceptions associated with the derivatives of the joint density of the inter-arrival times. E.g., for $i, j, k>0$

$$
\gamma_{k}^{-i,-j}=\alpha E^{-i} G^{k} E^{-j} \mathbb{I}=\alpha\left(-A_{0}\right)^{i}\left(\left(-A_{0}\right)^{-1} A_{1}\right)^{k}\left(-A_{0}\right)^{j} \mathbb{I}=\left.(-1)^{i+j-1} \frac{\partial^{i-1}}{\partial x_{0}^{i-1}} \frac{\partial^{j}}{\partial x_{k}^{j}} f\left(x_{0}, x_{k}\right)\right|_{x_{0}=x_{k}=0}
$$


The $M_{1}$ and $M_{2}$ matrices and (10) can also be used to obtain the relations of the $\gamma_{k_{2}, \ldots, k_{m}}^{i_{1}, i_{2}, \ldots, i_{m}}$ quantities for negative indexes.

For example based on the $r_{1}, r_{2}, r_{3}$ reduced moments and the $\gamma_{1}^{-1,-1}$ reduced joint moment of a $\operatorname{MEP}(2)$ we can compute the missing element of the basic moment set, the $\gamma_{1}^{1,1}$ reduced joint moment, using

$$
M_{1}(-1,-1,1)=\left[\begin{array}{ccc}
\gamma_{1}^{-1,-1} & \gamma^{-1} & \gamma^{0} \\
\gamma^{-1} & \gamma^{0} & \gamma^{1} \\
\gamma^{1,-1} & \gamma^{1} & \gamma^{2}
\end{array}\right] \text { and } M_{2}(1,-1,1)=\left[\begin{array}{ccc}
\gamma_{1}^{1,-1} & \gamma^{1} & \gamma_{1}^{1,1} \\
\gamma^{-1} & \gamma^{0} & \gamma^{1} \\
\gamma^{0} & \gamma^{1} & \gamma^{2}
\end{array}\right]
$$

such that $\operatorname{det}\left(M_{1}(-1,-1,1)\right)=0$ results $\gamma_{1}^{1,-1}$ and $\operatorname{det}\left(M_{2}(1,-1,1)\right)=0$ results $\gamma_{1}^{1,1}$.

\section{Conclusion}

This paper provides a methodology to investigate the relation of moments of ME distributions and MEPs. The presented results are also valid for PH distributions and MAPs as they are proper subsets of ME distributions and MEPs, respectively.

Based on this methodology we presented an algorithm to compute all possible reduced joint moments of MEPs based on a basic set of moments, referred to as basic moment set, without having any $\left(A_{0}, A_{1}\right)$ matrix representation of the process. Examples demonstrated, e.g., that

- there is more than one moment set that uniquely determines all possible moments of MEPs,

- an arbitrary set of $2 n$ moments does not always define a $\mathrm{ME}(\mathrm{n})$ distribution uniquely,

- it is hard to see the relation and the dependencies of the moments, but it is possible to construct a set of equations that describe these relations,

- the moments can be extended toward negative indices; the derivatives of the joint pdf of the interarrival times at zero carry similar information to the moments and they can define all possible moments of MEPs as well as the elements of the basic moment set.

In our future work we intend to apply this compact moment representation of MEPs in moment matching and fitting.

\section{References}

[1] G. Latouche and V. Ramaswami, Introduction to matrix analytic methods in stochastic modeling. SIAM, 1999

[2] M. Neuts, "A versatile markovian point process," Journal of Applied Probability, vol. 16, pp. 764779, 1979.

[3] M. Neuts, Matrix-Geometric Solutions in Stochastic Models. Baltimore, MD, USA: John Hopkins University Press, 1981. 
[4] S. Asmussen and M. Bladt, "Point processes with finite-dimensional conditional probabilities," Stochastic Processes and their Applications, vol. 82, pp. 127-142(16), July 1999.

[5] M. Fackrell, "Fitting with matrix-exponential distributions," Stochastic Models, vol. 21, pp. 377400, 2005.

[6] S. Asmussen and M. Bladt, "Renewal theory and queueing algorithms for matrix-exponential distributions," in Proc. 1st Int. Conference on Matrix-Analytic Methods in Stochastic Models, pp. 313-341, 1996.

[7] S. Asmussen and C. A. O'Cinneide, "Matrix-exponential distributions - distributions with a rational Laplace transform," in Encyclopedia of Statistical Sciences (S. Kotz and C. Read, eds.), (New York), pp. 435-440, John Wiley \& Sons, 1997.

[8] M. Bladt and M. Neuts, "Matrix-exponential distributions: Calculus and interpretations via flows," Commun. Statist.-Stochastic Models, 2003.

[9] K. Mitchell, K. Sohraby, A. van de Liefvoort, and J. Place, "Approximation models of wireless cellular networks using moment matching," in Proc. Conf. on Computer Communications (IEEE Infocom), pp. 189-197, 2000.

[10] A. van de Liefvoort, "The moment problem for continuous distributions," tech. rep., University of Missouri, WP-CM-1990-02, Kansas City, 1990.

[11] W. B. Gragg and A. Linquist, "On the partial realization problem," Linear Algebra and Applications, vol. 50, pp. 277-319, 1983.

[12] Q.-M. He and H. Zhang, "On matrix-exponential distributions," Advances in Applied Probability, vol. 39 , no. 1, pp. 271-292, 2007.

[13] K. Mitchell and A. van de Liefvoort, "Approximation models of feed-forward G/G/1/N queueing networks with correlated arrivals," Performance Evaluation, vol. 51, pp. 137-152, 2003.

[14] L. Lipsky, Queueing Theory: A linear algebraic approach. New York: MacMillan, 1992.

[15] M. Telek and A. Heindl, "Matching moments for acyclic discrete and continuous phase-type distributions of second order," International Journal of Simulation Systems, Science 83 Technology, vol. 3(3-4), pp. 47-57, dec. 2002. Special Issue on: Analytical \& Stochastic Modelling Techniques.

[16] L. Bodrog, A. Heindl, G. Horvath, and M. Telek, "A markovian canonical form of second-order matrix-exponential processes," European Journal of Operational Research, no. EOR 8488, 2007. 